\title{
Spin-flip and spin-wave excitations in arbitrarily polarized quantum Hall states
}

\author{
Sudhansu S. Mandalt \\ Department of Physics, Indian Institute of Science, Bangalore 560 012, India \\ and \\ Condensed Matter Theory Unit, Jawaharlal Nehru Center for Advanced Scientific Research, \\ Jakkur, Bangalore 560 064, India
}

\begin{abstract}
We study spin-flip and spin-wave excitations for arbitrarily polarized quantum Hall states by employing a fermionic Chern-Simons gauge theory in the low Zeeman energy limit. We show that the spin-flip correlation functions do not get renormalized by the fluctuations of Chern-Simons gauge field. As a consequence, the excitations for a given integer quantum Hall state are identical to fractional quantum Hall states in the lowest Landau level having the same numerator equal to the integer quantum Hall state. Fully and partially polarized states possess only spin-wave excitations while spin-flip excitations are possible for all states, irrespective of their polarizations.

PACS number(s): 73.40.Hm, 73.20.Mf
\end{abstract}

Typeset using REVTEX 


\section{INTRODUCTION}

By now the composite fermion model is well established in fractional quantum Hall effect. Proposed originally by Jain [四] in order to describe the fractional quantum Hall states (QHS), it was field theoretically developed by Lopez and Fradkin [2], and Halperin, Lee, and Read [3] who studied the model near the filling fraction $\nu=1 / 2$. Many experiments [4] have also confirmed the existence of composite fermions. Subsequently, the composite fermion (CF) picture has been extended to describe QHS with arbitrary polarization, by Mandal and Ravishankar [15]. The model, which we shall call the doublet model, employs a doublet of Chern-Simons (CS) gauge fields corresponding to two spin degrees of freedom. Mandal and Ravishankar have shown that almost all the observed quantum Hall states can be described by the mean field (MF) of CS gauge fields. They have also studied the fluctuations about MF configurations. They have further studied [16] charge density and spin density excitations $\left(\delta S_{z}=0\right)$ for these arbitrarily polarized QHS (APQHS) using time dependent Hartree-Fock approximation (TDHFA) for the Coulomb interaction between CF's.

In this paper, we further study the spin flip and spin wave excitations $\left(\delta S_{z}= \pm 1\right)$ for APQHS. Stein et al [17] were the first to observe experimentally the electron spin resonance corresponding to spin wave excitations in odd integer QHS. This type of excitations is possible only for odd integer QHS; for then, if the Lande $g$ factor is small, one spin state in the topmost filled Landau level (LL) will be filled, leaving the other closeby spin state unoccupied. Spin flip and spin wave excitations in these systems were first studied theoretically by Kallin and Halperin [18. They employed a diagrammatic approach which is equivalent to TDHFA for the Coulomb interactions between electrons. Subsequently, Longo and Kallin [19] extended the analysis of spin flip excitations to partial filling factors. They speculated that the change in the energy of excitations due to the Coulomb interactions is

simply the filling factor times the corresponding value for fully filled lowest LL; the lowest energy required for spin flip excitations is the cyclotron energy. In fact, we show below that the change in the energy of excitations due to the Coulomb interactions between composite 
fermions for a given fractional filling factor in the lowest LL is equal to the corresponding value of energy for the corresponding value of fully filled integer state. In other words, all the QHS in the lowest LL which have the same numerator have the same spin flip excitation energy. Further, the lowest mode corresponding to the spin flip excitations is the effective cyclotron energy of the CF, in consistency with what we expect from CF picture.

We show that the CS field fluctuations do not contribute to the spin flip correlation functions (SFCF). The latter hence may be determined exactly (without making an expansion in wave vector). Moreover, it is the same for all the states which have identical number of fully filled effective LL. We find that the Coulomb interaction between CF's produces an additional gap for spin flip excitations, in general. On the other hand, spin wave modes are gapless as $g \rightarrow 0$, as required by Larmor's theorem.

The plan of the paper is as follows. In the next section, we briefly reintroduce the doublet model and then present the formalism for determining SFCF corresponding to spin density excitations (SDE) with $\delta S_{z}= \pm 1$. In section III, we evaluate SFCF in the TDHFA by a diagrammatic approach for the Coulomb interaction between the CF. In section IV, we present spin flip and spin wave excitations for APQHS. Section V is devoted to a summary and discussion to the paper.

\section{THE FORMALISM}

To present it briefly, consider the 'doublet Lagrangian' [15]

$$
\begin{aligned}
\mathcal{L}= & \psi_{\uparrow}^{*} \mathcal{D}\left(a_{\mu}^{+}+a_{\mu}^{-}\right) \psi_{\uparrow}+\psi_{\downarrow}^{*} \mathcal{D}\left(a_{\mu}^{+}-a_{\mu}^{-}\right) \psi_{\downarrow}+\frac{\theta_{+}}{2} a_{\mu}^{+} \epsilon^{\mu \nu \lambda} \partial_{\nu} a_{\lambda}^{+} \\
& +\frac{\theta_{-}}{2} a_{\mu}^{-} \epsilon^{\mu \nu \lambda} \partial_{\nu} a_{\lambda}^{-}-e A_{0}^{\text {in }} \rho+e \psi^{\dagger}\left(\sigma_{+} h^{+}+\sigma_{-} h^{-}\right) \psi \\
& +\frac{1}{2} \int d^{3} x^{\prime} A_{0}^{\text {in }}(x) V^{-1}\left(x-x^{\prime}\right) A_{0}^{\text {in }}\left(x^{\prime}\right)
\end{aligned}
$$

where we have introduced additional sources $h^{ \pm}$which can flip spin. Here $\psi \equiv\left(\psi_{\uparrow}, \psi_{\downarrow}\right)$ is the doublet of fermionic fields where $\uparrow(\downarrow)$ represents spin up(down). We define $\mathcal{D}\left(a_{\mu}\right)$ as

$$
\mathcal{D}\left(a_{\mu}\right)=i D_{0}+\left(1 / 2 m^{*}\right) D_{k}^{2}+\mu+(g / 2) \mu_{B} B \sigma
$$


with $D_{\mu}=\partial_{\mu}-i e\left(A_{\mu}+a_{\mu}+A_{0}^{\text {in }} \delta_{\mu 0}\right)$, where $A_{\mu}$ is the external electromagnetic field. $a_{\mu}^{ \pm}$ are the CS gauge fields which interact in phase with spin up particles while they interact out of phase with spin down particles. The field $A_{0}^{\text {in }}$ is internal scalar potential. The mean particle density $\rho$ is held fixed by the chemical potential $\mu . m^{*}$ is the effective mass of the particles. The Zeeman term includes applied magnetic field [20]. $\mu_{B}$ is the Bohr magneton and $\sigma=+1(-1)$ for spin-up (-down) particles. The potential for interactions between $\mathrm{CF}$ is considered to be Coulombic, i.e., $V(r)=e^{2} / \epsilon r$, where $\epsilon$ is the background dielectric constant of the system. $\sigma_{ \pm}=\frac{1}{2}\left(\sigma_{x} \pm i \sigma_{y}\right)$ are the spin raising and lowering operators respectively.

\section{A. Mean field results (a brief resume)}

As in Ref. [15], we parametrize $\theta_{ \pm}=\left(e^{2} / 2 \pi\right)\left(1 / s_{ \pm}\right)$and set $s_{-}=0$ and $s_{+}=2 s$ (even integer). For this choice, the field $a_{\mu}^{-}$provides a vanishing mean magnetic field $\left\langle b^{-}\right\rangle$and it essentially decouples. The CF picture is enforced by the choice $s_{+}=2 s$. Thus, in the MF ansatz, the CS magnetic field produced by the particle is $\left\langle b^{+}\right\rangle=-e \rho / \theta_{+}$. The mean magnetic field experienced by the particles, irrespective of their spin, is given by $\bar{B}=B+\left\langle b^{+}\right\rangle$. Let $p_{\uparrow}\left(p_{\downarrow}\right)$ be the number of LL, which are formed by effective field $\bar{B}$, filled by spin up (down) particles. This leads to the actual filling fraction and spin density [15]

$$
\nu=\frac{p_{\uparrow}+p_{\downarrow}}{2 s\left(p_{\uparrow}+p_{\downarrow}\right)+1}, \Delta \rho=\rho\left(\frac{p_{\uparrow}-p_{\downarrow}}{p_{\uparrow}+p_{\downarrow}}\right) .
$$

Note that $p_{\uparrow}$ and $p_{\downarrow}$ can be negative integers as well in which case $\bar{B}$ is antiparallel to $B$. The effective cyclotron frequency $\bar{\omega}_{c}$ is related to the actual cyclotron frequency $\omega_{c}=\frac{e}{m^{*}} B$ by $\omega_{c}=\bar{\omega}_{c}\left[2 s\left(p_{\uparrow}+p_{\downarrow}\right)+1\right]$. For unpolarized QHS, $p_{\uparrow}=p_{\downarrow}=p$ (say) and therefore the states with filling fraction $\nu=2 p /(4 s p+1)$ are spin unpolarized in the limit of small Zeeman energy. In this limit, $p_{\uparrow}=p_{\downarrow}+1$ for partially polarized states with $\Delta \rho / \rho=1 /\left(p_{\uparrow}+p_{\downarrow}\right)$. Fully polarized Laughlin states are obtained for $p_{\uparrow}=1, p_{\downarrow}=0$. The integer QHS's correspond to the choice $s=0$ (i.e., $\theta_{+}=\infty$ ), in which case mean CS magnetic field is zero. 


\section{B. Effective action}

Employing the above MF ansatz along with vanishing mean electric fields $\left\langle\mathbf{e}^{ \pm}\right\rangle$and $\left\langle A_{0}^{\text {in }}\right\rangle=0$, we then evaluate the one-loop effective action for the gauge fields and probes to be

$$
\begin{aligned}
S_{\mathrm{eff}}= & -\frac{1}{2} \int d^{3} x \int d^{3} x^{\prime}\left[\left(a_{\mu}^{+}+a_{\mu}^{-}+A_{0}^{\mathrm{in}} \delta_{\mu 0}\right)(x) \Pi_{\uparrow}^{\mu \nu}\left(x, x^{\prime}\right)\left(a_{\nu}^{+}+a_{\nu}^{-}+A_{0}^{\mathrm{in}} \delta_{\nu 0}\right)\left(x^{\prime}\right)\right. \\
& +\left(a_{\mu}^{+}-a_{\mu}^{-}+A_{0}^{\mathrm{in}} \delta_{\mu 0}\right)(x) \Pi_{\downarrow}^{\mu \nu}\left(x, x^{\prime}\right)\left(a_{\nu}^{+}+a_{\nu}^{-}+A_{0}^{\mathrm{in}} \delta_{\nu 0}\right)\left(x^{\prime}\right) \\
& -A_{0}^{\mathrm{in}}(x) V^{-1}\left(x-x^{\prime}\right) A_{0}^{\mathrm{in}}\left(x^{\prime}\right)+2 h^{a}(x) \Gamma_{\uparrow}^{a \mu}\left(x, x^{\prime}\right)\left(a_{\mu}^{+}+a_{\mu}^{-}+A_{0}^{\mathrm{in}} \delta_{\mu 0}\right)\left(x^{\prime}\right) \\
& \left.+2 h^{a}(x) \Gamma_{\downarrow}^{a \mu}\left(x, x^{\prime}\right)\left(a_{\mu}^{+}-a_{\mu}^{-}+A_{0}^{\mathrm{in}} \delta_{\mu 0}\right)\left(x^{\prime}\right)+h_{a}(x) \chi^{a b}\left(x, x^{\prime}\right) h_{b}\left(x^{\prime}\right)\right] \\
& +\frac{1}{2} \int d^{3} x\left\{\theta_{+} \epsilon^{\mu \nu \lambda} a_{\mu}^{+} \partial_{\nu} a_{\lambda}^{+}+\theta_{-} \epsilon^{\mu \nu \lambda} a_{\mu}^{-} \partial_{\nu} a_{\lambda}^{-}\right\} \quad ; \quad a, b= \pm .
\end{aligned}
$$

Here $a_{\mu}^{ \pm}$and $A_{0}^{\text {in }}$ are fluctuating parts of the corresponding gauge fields. Note that we have kept $a_{\mu}^{-}$field in the effective action for the sake of completeness, although it decouples for the states (3) at hand. At the end of the calculation, however, the limit $\theta_{-}=\infty$ has to be taken. The correlation functions $\Pi_{r}^{\mu \nu}\left(x, x^{\prime}\right), \chi^{a b}\left(x, x^{\prime}\right)$, and $\Gamma_{r}^{a \mu}\left(x, x^{\prime}\right)$ have to be evaluated at the prescribed MF configuration. Their explicit forms are as follows:

$$
\begin{aligned}
& \Pi_{r}^{\mu \nu}\left(x, x^{\prime}\right)=-i\left\langle j_{r}^{\mu}(x) j_{r}^{\nu}\left(x^{\prime}\right\rangle_{C}-\left\langle\frac{\delta j_{r}^{\mu}(x)}{\delta \mathcal{A}_{\nu}\left(x^{\prime}\right)}\right\rangle\right. \\
& \chi^{a b}\left(x, x^{\prime}\right)=-i\left\langle j^{a}(x) j^{b}\left(x^{\prime}\right)\right\rangle_{C} \\
& \Gamma_{r}^{a \mu}\left(x, x^{\prime}\right)=-i\left\langle j^{a}(x) j_{r}^{\mu}\left(x^{\prime}\right)\right\rangle_{C}
\end{aligned}
$$

Here $\langle\cdots\rangle$ represents the expectation value in the ground state of the system. $\langle\cdots\rangle_{C}$ corresponds to the connected diagrams which contribute to the expectation value. $\mathcal{A}_{\mu}$ represents the sum of all the gauge fields. The current operators in Eqs. (5-7) are given by

$$
\begin{aligned}
j_{r}^{0}(x) & =e \psi_{r}^{*} \psi_{r} \\
j_{r}^{k}(x) & =e \frac{e}{2 m^{*}}\left[\psi_{r}^{*} D^{k} \psi_{r}-\left(D^{k *} \psi_{r}^{*}\right) \psi_{r}\right], \\
j^{a}(x) & =e \psi^{\dagger} \sigma_{a} \psi
\end{aligned}
$$

It is easy to see that 


$$
\Gamma_{r}^{a \mu}\left(x, x^{\prime}\right) \equiv 0
$$

since $\left\langle\sigma_{a}\right\rangle \equiv 0$. Therefore, the terms in Eq. (四) corresponding to the probe $h_{a}$ completely decouple from the fluctuation of the gauge fields. In other words, gauge field fluctuations do not change the correlation $\chi^{a b}\left(x, x^{\prime}\right)$. Further, $\chi^{++}\left(x, x^{\prime}\right)=\chi^{--}\left(x, x^{\prime}\right) \equiv 0$ since $\left\langle\sigma_{+}^{2}\right\rangle=$ $\left\langle\sigma_{-}^{2}\right\rangle \equiv 0$. We thus obtain

$$
S_{\mathrm{eff}}\left[h^{+}, h^{-}\right]=-\frac{1}{2} \int d^{3} x \int d^{3} x^{\prime}\left[h^{+}(x) \chi^{+-}\left(x, x^{\prime}\right) h^{-}\left(x^{\prime}\right)+h^{-}(x) \chi^{-+}\left(x, x^{\prime}\right) h^{+}\left(x^{\prime}\right)\right] .
$$

$\chi^{+-}\left(x, x^{\prime}\right) \equiv \delta^{2} S_{\text {eff }} / \delta h^{+}(x) \delta h^{-}\left(x^{\prime}\right)$ is the spin correlation function where a particle of up spin is destroyed at the point $x^{\prime}$ and a particle of spin down is created at the point $x$. In other words, this is the correlation function for producing a spin up quasihole at the point $x^{\prime}$ and a spin down quasiparticle at the point $x$. Similarly, $\chi^{-+}\left(x, x^{\prime}\right)$ represents the spin correlation function for producing a spin down quasihole at the point $x^{\prime}$ and a spin up quasiparticle at the point $x$. These correlation functions are the response functions for spin density excitation $\left(\delta S_{z}= \pm 1\right)$ which we shall evaluate below.

\section{RESPONSE FUNCTIONS}

In terms of the single particle Green function

$$
G\left(x, x^{\prime}\right)=-i\left\langle T \psi(x) \psi^{\dagger}\left(x^{\prime}\right)\right\rangle
$$

the linear response functions can be written as

$$
\begin{aligned}
& \chi^{+-}\left(x, x^{\prime}\right)=i e^{2} \operatorname{Tr}\left[\sigma_{+} G\left(x, x^{\prime}\right) \sigma_{-} G\left(x^{\prime}, x\right)\right], \\
& \chi^{-+}\left(x, x^{\prime}\right)=i e^{2} \operatorname{Tr}\left[\sigma_{-} G\left(x, x^{\prime}\right) \sigma_{+} G\left(x^{\prime}, x\right)\right] .
\end{aligned}
$$

Let $G_{0}$ be the single particle Green's function evaluated by switching off the Coulomb interaction. Then $\chi_{0}^{+-}$and $\chi_{0}^{-+}$that emerge are in pure random phase approximation (RPA). We shall go beyond RPA and determine $\chi^{+-}$and $\chi^{-+}$in the TDHFA. 
We now determine the response functions (in momentum space) in the TDHFA as a generalization to the RPA. Here we employ the diagrammatic approach which was developed by Kallin and Halperin [18]. Recall that in TDHFA, the Green's functions are Hartree Fock Green's functions and incorporate self energy due to the Coulomb interactions. In this approximation, only those diagrams with one exciton present at a time are considered. In other words, the Coulomb energy $e^{2} / \epsilon l_{0}$ is taken to be smaller than $\bar{\omega}_{c}$, where $l_{0}=(e \bar{B})^{-1 / 2}$ is the effective magnetic length of the system and $\epsilon$ is the background dielectric constant. This assumption is, therefore, not valid at or near $\nu=1 / 2 s$. In other words, the condition on the validity of our assumption is $e^{3 / 2} m^{*} / \epsilon \ll \sqrt{\bar{B}}$.

The TDHFA response functions are determined in the appendix A. They are

$$
\begin{aligned}
\chi^{+-}(\omega, \mathbf{q})= & \frac{e^{2}}{4 \pi} \mathbf{q}^{2} e^{-\overline{\mathbf{q}}^{2}} \\
& \times\left[\sum_{n_{2}<p_{\uparrow}} \sum_{n_{1} \geq p_{\downarrow}}\left(\frac{n_{2} !}{n_{1} !}\right) \frac{\left(\overline{\mathbf{q}}^{2}\right)^{n_{1}-n_{2}-1}\left\{L_{n_{2}}^{n_{1}-n_{2}}\left(\overline{\mathbf{q}}^{2}\right)\right\}^{2}}{\omega-\left(\epsilon_{n_{1}}^{\downarrow}-\epsilon_{n_{2}}^{\uparrow}\right)-E_{n_{1} n_{2}}^{\downarrow \uparrow}+\tilde{V}_{n_{1} n_{2} n_{2} n_{1}}^{(1)}(q)+i \eta}\right. \\
& \left.-\sum_{n_{2}<p_{\downarrow}} \sum_{n_{1} \geq p_{\uparrow}}\left(\frac{n_{2} !}{n_{1} !}\right) \frac{\left(\overline{\mathbf{q}}^{2}\right)^{n_{1}-n_{2}-1}\left\{L_{n_{2}}^{n_{1}-n_{2}}\left(\overline{\mathbf{q}}^{2}\right)\right\}^{2}}{\omega+\left(\epsilon_{n_{1}}^{\uparrow}-\epsilon_{n_{2}}^{\downarrow}\right)+E_{n_{1} n_{2}}^{\uparrow \downarrow}-\tilde{V}_{n_{1} n_{2} n_{2} n_{1}}^{(1)}(q)-i \eta}\right], \\
\chi^{-+}(\omega, \mathbf{q})= & \frac{e^{2}}{4 \pi} \mathbf{q}^{2} e^{-\overline{\mathbf{q}}^{2}} \\
& \times \sum_{n_{2}<p_{\downarrow}} \sum_{n_{1} \geq p_{\uparrow}}\left(\frac{n_{2} !}{n_{1} !}\right) \frac{\left(\overline{\mathbf{q}}^{2}\right)^{n_{1}-n_{2}-1}\left\{L_{n_{2}}^{n_{1}-n_{2}}\left(\overline{\mathbf{q}}^{2}\right)\right\}^{2}}{\omega-\left(\epsilon_{n_{1}}^{\uparrow}-\epsilon_{n_{2}}^{\downarrow}\right)-E_{n_{1} n_{2}}^{\uparrow \downarrow}+\tilde{V}_{n_{1} n_{2} n_{2} n_{1}}^{(1)}(q)+i \eta} \\
& \left.-\sum_{n_{2}<p_{\uparrow}} \sum_{n_{1} \geq p_{\downarrow}}\left(\frac{n_{2} !}{n_{1} !}\right) \frac{\left(\overline{\mathbf{q}}^{2}\right)^{n_{1}-n_{2}-1}\left\{L_{n_{2}}^{n_{1}-n_{2}}\left(\overline{\mathbf{q}}^{2}\right)\right\}^{2}}{\omega+\left(\epsilon_{n_{1}}^{\downarrow}-\epsilon_{n_{2}}^{\uparrow}\right)+E_{n_{1} n_{2}}^{\downarrow \uparrow}-\tilde{V}_{n_{1} n_{2} n_{2} n_{1}}^{(1)}(q)-i \eta}\right],
\end{aligned}
$$

where $\overline{\mathbf{q}}^{2}=\mathbf{q}^{2} l_{0}^{2} / 2$ and $n_{1}, n_{2}$ represent the indices of the LL formed by effective magnetic field $\bar{B}$. Here $\epsilon_{n}^{r}=(n+1 / 2) \bar{\omega}_{c}-(1 / 2) g \mu_{B} B \sigma$ is the energy of $n$th LL with spin index r. $\sigma=+1(-1)$ for up (down) states. $E_{n_{1} n_{2}}^{r^{\prime}}=\Sigma_{n_{1}}^{r}-\Sigma_{n_{2}}^{r^{\prime}}$ represents the exchange energy, i.e., the difference in self energy of particles in two different LL with unequal spin indices. $\Sigma_{n}^{r}$ is independent of momentum $q$, and is given by Eq. (A2). The interaction between an 
excited particle and hole (i.e., the ladder diagrams) is represented by the matrix element $\tilde{V}_{n_{1} n_{2} n_{2} n_{1}}^{(1)}(q)$ which is expressed in Eq. (A12). We note that the bubble diagrams in which a particle-hole pair recombines to form another particle-hole pair do not contribute to the response functions considered here. This is because particle and hole possess different spin.

In obtaining Eqs. (16) and (17), we have included all the contributions up to order $e^{2} / \epsilon l_{0}$ and so the form factors are essentially exact in the strong effective magnetic field limit. In the absence of Coulomb interaction, $\Sigma_{n}^{r}$ and $\tilde{V}_{n_{1} n_{2} n_{2} n_{1}}^{(1)}(q)$ are zero and the response functions acquire their pure RPA form. We shall see below that the coulomb interaction between composite fermions plays a decisive role in the excitations considered that we are interested in.

\section{EXCITATIONS}

As we have seen above, the response functions $\chi^{+-}$and $\chi^{-+}$remain unchanged by the fluctuation of the gauge fields. They depend only on the mean effective magnetic field. Therefore, the corresponding SDE depend solely on the effective magnetic length $l_{0}$ which is related to the actual magnetic length of the system $l=(e B)^{-1 / 2}$, via $l_{0}=\left(\left|p_{\uparrow}+p_{\downarrow}\right| / \nu\right)^{1 / 2} l$. The excitations for the fractional states with $\nu=\left|p_{\uparrow}+p_{\downarrow}\right| /\left(2 s\left|p_{\uparrow}+p_{\downarrow}\right| \pm 1\right)$ are equivalent to that of integer states with $\nu=\left|p_{\uparrow}+p_{\downarrow}\right|$, since the states have same $l_{0}$.

The dispersion relation for the excitation of a spin down particle and a spin up hole is obtained from Eq. (16) as

$$
\omega=\left(n_{1}-n_{2}\right) \bar{\omega}_{c}+g \mu_{B} B+E_{n_{1} n_{2}}^{\downarrow \uparrow}-\tilde{V}_{n_{1} n_{2} n_{2} n_{1}}^{(1)}(q),
$$

with $n_{2}<p_{\uparrow}$ and $n_{1} \geq p_{\downarrow}$. In these SDE, the $z$ component of the spin changes as $\delta S_{z}=-1$. Similarly the SDE with a spin up particle and a spin up hole $\left(\delta S_{z}=+1\right)$ have dispersion relation (see Eq. (17))

$$
\omega=\left(n_{1}-n_{2}\right) \bar{\omega}_{c}-g \mu_{B} B+E_{n_{1} n_{2}}^{\uparrow \downarrow}-\tilde{V}_{n_{1} n_{2} n_{2} n_{1}}^{(1)}(q),
$$

with $n_{2}<p_{\downarrow}$ and $n_{1} \geq p_{\uparrow}$. 


\section{A. Spin-flip excitations}

In spin flip excitations, a particle changes the Landau level as well as flipping its spin. The excitation modes can have energy different from $\left(n_{1}-n_{2}\right) \bar{\omega}_{c}+g \mu_{B} B\left(\delta S_{z}\right)$ at $q=0$, since the Coulombic interaction changes the gap energy, in general. There are three types of ground state to consider: (i) fully polarized states $\left(p_{\uparrow}=1, p_{\downarrow}=0\right)$; (ii) unpolarized states $\left(p_{\uparrow}=p_{\downarrow}\right)$; and (iii) partially polarized states $\left(p_{\uparrow}=p_{\downarrow}+1\right)$. We discuss the spin-flip excitations of each of these ground states.

Case-I: For fully polarized states, the dispersion relation, corresponding to spin down particle and spin up hole excitations, from Eq. (18), is given by

$$
\begin{aligned}
\omega_{m}-m \bar{\omega}_{c}-g \mu_{B} B & =\Delta E_{m}(q) \\
& =E_{m 0}^{\downarrow \uparrow}-\tilde{V}_{m 00 m}^{(1)}(q),
\end{aligned}
$$

where $m$ is an integer. The changes in energy, due to the Coulomb interaction between the fermions, corresponding to the two lowest modes with $m=1$ and $m=2$, are respectively obtained as

$$
\begin{gathered}
\Delta E_{1}(q)=\frac{e^{2}}{\epsilon l_{0}} \frac{1}{2} \sqrt{\frac{\pi}{2}}\left\{2-e^{-\overline{\mathbf{q}}^{2} / 2}\left[\left(1+\overline{\mathbf{q}}^{2}\right) I_{0}\left(\frac{\overline{\mathbf{q}}^{2}}{2}\right)-\overline{\mathbf{q}}^{2} I_{1}\left(\frac{\overline{\mathbf{q}}^{2}}{2}\right)\right]\right\}, \\
\Delta E_{2}(q)=\frac{e^{2}}{\epsilon l_{0}} \frac{1}{8} \sqrt{\frac{\pi}{2}}\left\{8-e^{-\overline{\mathbf{q}}^{2} / 2}\left[\left(3+2 \overline{\mathbf{q}}^{2}+2 \overline{\mathbf{q}}^{4}\right) I_{0}\left(\frac{\overline{\mathbf{q}}^{2}}{2}\right)\right.\right. \\
\left.\left.-\left(4 \overline{\mathbf{q}}^{2}+2 \overline{\mathbf{q}}^{4}\right) I_{1}\left(\frac{\overline{\mathbf{q}}^{2}}{2}\right)\right]\right\} .
\end{gathered}
$$

Here $I_{0}$ and $I_{1}$ are the modified Bessel's functions whose integral representations are given by

$$
\begin{aligned}
I_{0}(z) & =\frac{1}{4 \pi} \int_{0}^{4 \pi} e^{-z \cos \theta} d \theta \\
\frac{1}{z} I_{1}(z) & =\frac{1}{4 \pi} \int_{0}^{4 \pi} e^{-z \cos \theta} \sin ^{2} \theta d \theta .
\end{aligned}
$$

These two dispersion relations are shown in Fig. 1. For very low $q l_{0}$, these energies behave as 


$$
\begin{aligned}
& \Delta E_{1}(q) \approx \frac{e^{2}}{\epsilon l_{0}} \frac{1}{2} \sqrt{\frac{\pi}{2}}\left[1-\frac{\overline{\mathbf{q}}^{2}}{2}\right], \\
& \Delta E_{2}(q) \approx \frac{e^{2}}{\epsilon l_{0}} \frac{1}{8} \sqrt{\frac{\pi}{2}}\left[5-\frac{\overline{\mathbf{q}}^{2}}{2}\right] .
\end{aligned}
$$

Thus the Coulomb interaction has changed the gap energy for these spin-flip excitations. At low momentum, the exchange in self energy dominates over the interaction energy between excited particle and hole pair. On the other hand, their contributions reverse for higher momentum. Thus the dispersion relations show a minima in their spectra (see Fig. 1). There is no mode corresponding to the dispersion relation (19), since the population of down spins is zero in the fully polarized ground state.

Note that the above dispersion relations of the spin-flip excitations hold for the Laughlin states with $\nu=\frac{1}{2 s+1}$ because they all have the same $l_{0}$. All these states acquire non vanishing gap energies due to the Coulomb interaction, contrary to the result of Kallin and Halperin 18 for $\nu=1$ state $(s=0)$. The reason for the mismatch between the two results is that they have calculated exchange self energy wrongly. This drawback was corrected by Longo and Kallin [19], who have further calculated dispersion relations using the single mode approximation (SMA) to obtain finite gap energies due to the Coulomb interaction in partially filled Landau levels. As we have seen, the present model allows for spin-flip excitation at $\bar{\omega}_{c}+g \mu_{B} B$, while the lowest spin-flip mode in the SMA is at $\omega_{c}+g \mu_{B} B$.

Case-II: For unpolarized QHS, equal number of LL filled by particles of up and down spins. Consider the simplest case $\left|p_{\uparrow}\right|=\left|p_{\downarrow}\right|=1$. The dispersion relations for the spin flip excitations with $\delta S_{z}=\mp 1$ are respectively given by

$$
\begin{aligned}
\omega_{m}-m \bar{\omega}_{c}-g \mu_{B} B & =\Delta E_{m}^{-} \\
& =E_{m 0}^{\downarrow \uparrow}-\tilde{V}_{m 00 m}^{(1)}(q), \\
\omega_{m}-m \bar{\omega}_{c}+g \mu_{B} B & =\Delta E_{m}^{+} \\
& =E_{m 0}^{\uparrow \downarrow}-\tilde{V}_{m 00 m}^{(1)}(q) .
\end{aligned}
$$

Here $\Delta E_{m}^{+}=\Delta E_{m}^{-}$as $E_{m 0}^{\downarrow \uparrow}=E_{m 0}^{\uparrow \downarrow}$ due to the equal population of both the spins. The change in energy due to the Coulomb interaction for the two lowest modes $(m=1,2)$ are given by 


$$
\begin{gathered}
\Delta E_{1}^{ \pm}(q)=\frac{e^{2}}{\epsilon l_{0}} \frac{1}{2} \sqrt{\frac{\pi}{2}}\left\{1-e^{-\overline{\mathbf{q}}^{2} / 2}\left[\left(1+\overline{\mathbf{q}}^{2}\right) I_{0}\left(\frac{\overline{\mathbf{q}}^{2}}{2}\right)-\overline{\mathbf{q}}^{2} I_{1}\left(\frac{\overline{\mathbf{q}}^{2}}{2}\right)\right]\right\}, \\
\Delta E_{2}^{ \pm}(q)=\frac{e^{2}}{\epsilon l_{0}} \frac{1}{8} \sqrt{\frac{\pi}{2}}\left\{5-e^{-\overline{\mathbf{q}}^{2} / 2}\left[\left(3+2 \overline{\mathbf{q}}^{2}+2 \overline{\mathbf{q}}^{4}\right) I_{0}\left(\frac{\overline{\mathbf{q}}^{2}}{2}\right)\right.\right. \\
\left.\left.-\left(4 \overline{\mathbf{q}}^{2}+2 \overline{\mathbf{q}}^{4}\right) I_{1}\left(\frac{\overline{\mathbf{q}}^{2}}{2}\right)\right]\right\}
\end{gathered}
$$

respectively. These are shown in Fig. 2. Note that the Coulomb interaction does not contribute to the energy of $m=1$ mode at $q l_{0}=0$. The unpolarized states with $\nu=$ $2 /(4 s \pm 1)$, i.e., the states with 2 as a numerator such as $2,2 / 3,2 / 5$ have similar spin flip excitations as above since they all have same $l_{0}$.

Case-III: Consider the simplest case $\left|p_{\uparrow}\right|=2$ and $\left|p_{\downarrow}\right|=1$ as an example of partially polarized QHS. The dispersion relation corresponding to $\delta S_{z}=+1$ excitations is given by

$$
\omega_{m}-m \bar{\omega}_{c}+g \mu_{B} B=\Delta E_{m}^{+}=E_{m 0}^{\uparrow \downarrow}-\tilde{V}_{m 00 m}^{(1)}(q)
$$

with $m>1$. Therefore there is no mode at $\bar{\omega}_{c}-g \mu_{B} B$ in this case. $\delta S_{z}=-1$ type of spin flip excitations may occur from any of the two filled LL by up spins. They have two classes of dispersion relations given by

$$
\begin{array}{r}
\omega_{m}-m \bar{\omega}_{c}-g \mu_{B} B=\Delta E_{m}^{(1)-}=E_{m 0}^{\downarrow \uparrow}-\tilde{V}_{m 00 m}^{(1)}(q) ; m>0, \\
\omega_{m}-(m-1) \bar{\omega}_{c}-g \mu_{B} B=\Delta E_{m}^{(2)-}=E_{m 1}^{\downarrow \uparrow}-\tilde{V}_{m 11 m}^{(1)}(q) ; m>1 .
\end{array}
$$

The energies $\Delta E_{1}^{(1)-}(q), \Delta E_{2}^{(1)-}(q)$, and $\Delta E_{2}^{(2)-}(q)$ are shown in Fig. 3. Figure 4 represents $\Delta E_{1}^{+}(q)$. The states with $\nu=3 /(6 s \pm 1)$, i.e., all the states with numerator 3 such as 3 , $3 / 5,3 / 7$ have the similar spin-flip excitations as shown above.

\section{B. Spin-wave excitations}

When the Fermi energy lies between two spin split levels in the same LL, the particles may be excited within the same LL by flipping the spin. This excitation is possible only in a ferromagnetic ground state. Therefore, fully polarized and partially polarized QHS are possible candidates to observe this kind of excitations. We shall see below that the Coulomb 
interactions do not change the spin-wave excitation energy at $q=0$, as required by Larmor's theorem. The long wave length spin wave becomes gapless at $g=0$.

Fully polarized states: Consider the fully polarized $\left(p_{\uparrow}=1, p_{\downarrow}=0\right)$ QHS first. The spin wave dispersion relation is obtained as

$$
\omega-g \mu_{B} B=\Delta E(q)=E_{00}^{\downarrow \uparrow}-\tilde{V}_{0000}^{(1)}(q) .
$$

The explicit form of $\Delta E(q)$ is given by

$$
\Delta E(q)=\frac{e^{2}}{\epsilon l_{0}} \sqrt{\frac{\pi}{2}}\left[1-e^{-\overline{\mathbf{q}}^{2} / 2} I_{0}\left(\frac{\overline{\mathbf{q}}^{2}}{2}\right)\right] .
$$

The dispersion energy $\Delta E(q)$ is shown in Fig. 5. The states $\nu=1$ and $\nu=1 /(2 s+1)$ follow the above dispersion relation in their spin wave excitations. At very low $q l_{0}$, the spin wave is quadratically dispersed as

$$
\omega=g \mu_{B} B+\frac{e^{2}}{\epsilon l_{0}} \frac{1}{2} \sqrt{\frac{\pi}{2}}\left(q l_{0}\right)^{2} .
$$

Nakajima and Aoki 21] also have numerically found spin wave excitations for these Laughlin states using CF picture. They used a reduced Haldane pseudo-potential 22] for CF's to carry out the computation in a spherical geometry. The energy cost to excite a spin down quasiparticle and a spin up quasihole is $\sqrt{\frac{\pi}{2}} \frac{e^{2}}{\frac{l_{0}}{2}}$. This excitation corresponds to $\overline{\mathbf{q}}^{2} \rightarrow \infty$ in Eq. (35).

Partially polarized states: For the simplest case of partially polarized QHS, $\left|p_{\uparrow}\right|=2$ and $\left|p_{\downarrow}\right|=1$. In this case, the spin wave dispersion relation is given by

$$
\omega-g \mu_{B} B=\Delta E=E_{11}^{\downarrow \uparrow}-\tilde{V}_{1111}^{(1)}(q)
$$

We find the dispersion energy to be

$$
\Delta E(q)=\frac{e^{2}}{\epsilon l_{0}} \frac{1}{4} \sqrt{\frac{\pi}{2}}\left[3-e^{-\overline{\mathbf{q}}^{2} / 2}\left\{\left(3-2 \overline{\mathbf{q}}^{2}+2 \overline{\mathbf{q}}^{4}\right) I_{0}\left(\frac{\overline{\mathbf{q}}^{2}}{2}\right)-2 \overline{\mathbf{q}}^{2} I_{1}\left(\frac{\overline{\mathbf{q}}^{2}}{2}\right)\right\}\right]
$$

which is shown in Fig. 6. The spin wave excitations of the states with $\nu=3$, and $\nu=$ $3 /(6 s \pm 1)$ such as $3,3 / 5,3 / 7$ have the above dispersion relation. At very low $q l_{0}$, this is again quadratically dispersed. The dispersion relation then is given by 


$$
\omega=g \mu_{B} B+\frac{e^{2}}{\epsilon l_{0}} \frac{7}{16} \sqrt{\frac{\pi}{2}}\left(q l_{0}\right)^{2} .
$$

In this case, the energy necessary to create a spin up quasiparticle and a spin down quasihole

pair is $\frac{3}{4} \sqrt{\frac{\pi}{2}} \frac{e^{2}}{\epsilon l_{0}}$ (see Eq. 38). Similarly other partially polarized QHS such as $\nu=5,5 / 9$, $5 / 11$ also possess spin wave excitations.

\section{SUMMARY AND DISCUSSIONS}

We have studied spin flip and spin wave excitations for arbitrarily polarized quantum Hall states in the time dependent Hartree-Fock approximation. We have employed fermionic Chern-Simons theory within the composite fermion picture. The spin flip correlation functions do not get renormalized by the fluctuations of Chern-Simons gauge field over its mean value which describes the ground state. That is they depend entirely on $p_{\uparrow}+p_{\downarrow}$ and the excitations are hence identical to the excitations in the corresponding integer state, same for the replacement magnetic length $l \rightarrow l_{0}$. We have shown that the Coulomb interaction between composite fermions produces an additional gap for spin flip excitations, in general. On the other hand, as a consequence of Larmor's theorem, neutral spin wave modes are gapless as $g \rightarrow 0$. There persists gap for creating a quasiparticle and quasihole pair. However, the lowest energy charged excitations in the ferromagnetic ground state of $\nu=1 /(2 s+1)$ are skyrmions 23] which are experimentally verified 24 26] at $\nu=1$. It has been found by $\mathrm{Wu}$ and Sondhi [27 that the odd integer states also possess skyrmion like excitations although they have higher energies compared to quasiparticle and quasihole pair excitations. It is now interesting to observe the occurrence of skyrmions in all the arbitrarily polarized quantum Hall states by a general frame work. An attempt in this line is in progress.

\section{ACKNOWLEDGMENTS}

I thank the anonymous referee of the paper in Ref. [16] for suggesting the problem which I have studied here. Many discussions with V. Ravishankar are gratefully acknowledged. 
Finally, I thank JNCASR, Bangalore, for financial assistance.

\section{APPENDIX A: RESPONSE FUNCTION IN TDHFA}

In this appendix, we shall evaluate $\chi^{a b}$ in TDHFA. We adopt the diagrammatic method that was developed by Kallin and Halperin [18].

The single particle Hartree-Fock Green's function is given by

$$
G_{n}^{r}(\omega)=\frac{1}{\omega-\epsilon_{n}^{r}-\Sigma_{n}^{r}+i \delta(n, r)}
$$

where $\delta(n, r)=0^{+}$for $n<p_{r}$ and $\delta(n, r)=0^{-}$for $n \geq p_{r} . G_{n}^{r}$ is diagrammatically shown in Fig. 7. In the strong field approximation (the effective field $\bar{B}$ here), the self energy, which is diagrammatically shown in Fig. 7 , is given by

$$
\Sigma_{n}^{r}=-\int \frac{d^{2} r}{2 \pi l_{0}^{2}} V(r) e^{-r^{2} / 2 l_{0}^{2}} L_{p_{r}-1}^{1}\left(\frac{r^{2}}{2 l_{0}^{2}}\right) L_{n}^{0}\left(\frac{r^{2}}{2 l_{0}^{2}}\right)
$$

where $V(r)=e^{2} / \epsilon r$ is the Coulomb potential. Note that the same index $r$ stands for both spin and spatial coordinates. The associated Laguerre polynomial is given by

$$
L_{n}^{m}(x)=\frac{1}{n !} e^{x} x^{-m} \frac{d^{n}}{d x^{n}}\left(e^{-x} x^{n+m}\right)
$$

The response function $\chi^{a b}(\omega \mathbf{q})$ may be expressed as (see Fig.8(a))

$$
\chi^{a b}(\omega, \mathbf{q})=\frac{e^{2}}{2 \pi l_{0}^{2}} \sum_{n_{1}, n_{2}} \sum_{r_{1}, r_{2}} M_{n_{1} n_{2}}(\mathbf{q}) D_{n_{1} n_{2}}^{r_{1} r_{2}}(\omega) \Gamma_{n_{1} n_{2}}^{r_{1} r_{2}}(\omega, \mathbf{q})\left\langle r_{1}\left|\sigma_{a}\right| r_{2}\right\rangle\left\langle r_{2}\left|\sigma_{b}\right| r_{1}\right\rangle
$$

Here the matrix element (contribution of the left vertex of Fig. 8(a)) is obtained as

$$
M_{n_{1} n_{2}}(\mathbf{q})=\left(\frac{2^{n_{2}} n_{2} !}{2^{n_{1}} n_{1} !}\right)^{1 / 2} e^{-\overline{\mathbf{q}}^{2} / 2}\left[\left(q_{x}+i q_{y}\right) l_{0}\right]^{n_{1}-n_{2}} L_{n_{2}}^{n_{1}-n_{2}}\left(\overline{\mathbf{q}}^{2}\right) .
$$

The two-particle propagator (see Fig. 8(a)) is given by

$$
\begin{aligned}
& D_{n_{1} n_{2}}^{r_{1} r_{2}}(\omega)=\int \frac{d \omega^{\prime}}{2 \pi i} G_{n_{1}}^{r_{1}}\left(\omega^{\prime}\right) G_{n_{2}}^{r_{2}}\left(\omega+\omega^{\prime}\right) \\
= & {\left[\frac{\theta\left(p_{r_{1}}-n_{1}\right) \theta\left(n_{2}-p_{r_{2}}-1\right)}{\omega-\left(\epsilon_{n_{1}}^{r_{1}}-\epsilon_{n_{2}}^{r_{2}}\right)-\left(\Sigma_{n_{1}}^{r_{1}}-\Sigma_{n_{2}}^{r_{2}}\right)+i \eta}-\frac{\theta\left(p_{r_{2}}-n_{2}\right) \theta\left(n_{1}-p_{r_{1}}-1\right)}{\omega-\left(\epsilon_{n_{1}}^{r_{1}}-\epsilon_{n_{2}}^{r_{2}}\right)-\left(\sum_{n_{1}}^{r_{1}}-\Sigma_{n_{2}}^{r_{2}}\right)-i \eta}\right], }
\end{aligned}
$$


where the function $\theta(x)$ is defined as

$$
\theta(x) \equiv\left\{\begin{array}{l}
1 \text { for } x>0 \\
0 \text { for } x<0
\end{array}\right.
$$

Finally, $\Gamma_{n_{1} n_{2}}^{r_{1} r_{2}}$ which is the corrected vertex (due to Coulomb interaction between the particle and hole pair) function is diagrammatically shown in Fig. 8(b). Here we have assumed that only a single exciton is present at a time. In other words, $e^{2} / \epsilon l_{0} \ll \bar{\omega}_{c}$.

Now if we define

$$
\Phi_{n_{1} n_{2}}^{r_{1} r_{2}}(\omega, \mathbf{q}) \equiv D_{n_{1} n_{2}}^{r_{1} r_{2}}(\omega) \Gamma_{n_{1} n_{2}}^{r_{1} r_{2}}(\omega, \mathbf{q})
$$

then the response function can be written as

$$
\chi^{a b}(\omega, \mathbf{q})=\frac{e^{2}}{2 \pi l_{0}^{2}} \sum_{r_{1}, r_{2}} \sum_{n_{1}, n_{2}} M_{n_{1} n_{2}}(\mathbf{q}) \Phi_{n_{1} n_{2}}^{r_{1} r_{2}}(\omega, \mathbf{q})\left\langle r_{1}\left|\sigma_{a}\right| r_{2}\right\rangle\left\langle r_{2}\left|\sigma_{b}\right| r_{1}\right\rangle,
$$

where $\Phi_{n_{1} n_{2}}^{r_{1} r_{2}}(\omega, \mathbf{q})$ satisfies the matrix equation (independent of spin indices),

$$
\sum_{n_{3}, n_{4}} \sum_{r_{3}, r_{4}}\left[\delta_{n_{1}, n_{3}} \delta_{n_{2}, n_{4}} \delta_{r_{1}, r_{3}} \delta_{r_{2}, r_{4}}\left[\{D(\omega)\}^{-1}\right]_{n_{3} n_{4}}^{r_{3} r_{4}}-\delta_{r_{1}, r_{3}} \delta_{r_{2}, r_{4}} \tilde{V}_{n_{1} n_{4} n_{2} n_{3}}^{(1)}(\mathbf{q})\right] \Phi_{n_{3} n_{4}}^{r_{3} r_{4}}=M_{n_{1} n_{2}}^{*}(\mathbf{q}) \text {. }
$$

Diagrammatically, $M_{n_{1} n_{2}}^{*}(\mathbf{q})$ is given by the first diagram of Fig. 8(b). The interaction between an excited electron and hole is represented by the ladder diagrams in the second diagram of Fig. 8(b). The corresponding matrix element due to to Coulomb interaction is given by

$$
\begin{aligned}
\tilde{V}_{n_{1} n_{4} n_{2} n_{3}}^{(1)}(\mathbf{q}) & =\left(\frac{2^{n_{4}} 2^{n_{2}} n_{4} ! n_{2} !}{2^{n_{1}} 2^{n_{3}} n_{1} ! n_{3} !}\right)^{1 / 2} \int \frac{d^{2} r}{2 \pi l_{0}^{2}} V\left(\mathbf{r}-l_{0}^{2} \mathbf{q} \times \hat{z}\right) e^{-r^{2} / 2 l_{0}^{2}} \\
& \times\left[\frac{x+i y}{l_{0}}\right]^{n_{1}-n_{2}}\left[\frac{x-i y}{l_{0}}\right]^{n_{3}-n_{4}} L_{n_{2}}^{n_{1}-n_{2}}\left(\frac{r^{2}}{2 l_{0}^{2}}\right) L_{n_{4}}^{n_{3}-n_{4}}\left(\frac{r^{2}}{2 l_{0}^{2}}\right),
\end{aligned}
$$

for $n_{2} \leq n_{1}$ and $n_{4} \leq n_{3}$. If $n_{2}>n_{1}$, then $n_{2}$ and $n_{1}$ in the right side of Eq. (A12) are interchanged; and similarly if $n_{4}>n_{3}$, then $n_{3}$ and $n_{4}$ are interchanged.

Solving $\Phi_{n_{1} n_{2}}^{r_{1} r_{2}}(\omega, \mathbf{q})$ from Eq. (A11), we substitute it in Eq. (A10) to obtain the response function, 


$$
\begin{aligned}
& \chi^{a b}(\omega, \mathbf{q})=\frac{e^{2}}{2 \pi l_{0}^{2}} \sum_{r_{1}} \sum_{r_{2}}\left\langle r_{1}\left|\sigma_{a}\right| r_{2}\right\rangle\left\langle r_{2}\left|\sigma_{b}\right| r_{1}\right\rangle \\
\times & {\left[\sum_{n_{2}<p_{r_{1}}} \sum_{n_{1} \geq p_{r_{2}}} \frac{\left|M_{n_{1} n_{2}}(\mathbf{q})\right|^{2}}{\omega-\left(\epsilon_{n_{1}}^{r_{2}}-\epsilon_{n_{2}}^{r_{1}}\right)-\left(\sum_{n_{1}}^{r_{2}}-\sum_{n_{2}}^{r_{1}}\right)+\tilde{V}_{n_{1} n_{2} n_{2} n_{1}}^{(1)}(\mathbf{q})+i \eta}\right.} \\
& \left.-\sum_{n_{2}<p_{r_{2}}} \sum_{n_{1} \geq p_{r_{1}}} \frac{\left|M_{n_{1} n_{2}}(\mathbf{q})\right|^{2}}{\omega+\left(\epsilon_{n_{1}}^{r_{1}}-\epsilon_{n_{2}}^{r_{2}}\right)+\left(\sum_{n_{1}}^{r_{1}}-\sum_{n_{2}}^{r_{2}}\right)-\tilde{V}_{n_{1} n_{2} n_{2} n_{1}}^{(1)}(\mathbf{q})-i \eta}\right],
\end{aligned}
$$

where $\sigma_{a}\left(\sigma_{b}\right)$ creates spin state $r_{1}\left(r_{2}\right)$ destroying spin state $r_{2}\left(r_{1}\right)$. Therefore, $r_{1} \neq r_{2}$. Note that the bubble diagrams (see the third diagram of Fig. 8(b)) do not contribute to the spin-flip response function. This is because in this response function, particle and hole possess different spins, and the Coulomb interaction can not flip the spin. 


\section{REFERENCES}

* Electronic address: ssman@physics.iisc.ernet.in

[1] J. K. Jain, Phys. Rev. Lett. 63, 199 (1989).

[2] A. Lopez and E. Fradkin, Phys. Rev. B 44, 5246 (1991).

[3] B. I. Halperin, P. A. Lee, and N. Read, Phys. Rev. B 47, 7312 (1993).

[4] R. R. Du, H. L. Stormer, D. C. Tsui, L. N. Pfeiffer, and K. W. West, Phys. Rev. Lett. 70, 29444 (1993).

[5] R. L. Willett, R. R. Ruel, M. A. Paalanen, K. W. West, and L. N. Pfeiffer, Phys. Rev. B 47, 7344 (1993); ibid. 71, 3846 (1993).

[6] W. Kang, H. L. Stormer, L. N. Pfeiffer, K. W. Baldwin, and K. W. West, Phys. Rev. Lett. 71, 3850 (1993).

[7] D. R. Leadley, H. L. Stormer, C. T. Foxon, and J. J. Harris, Phys. Rev. Lett. 72, 1906 (1994).

[8] R. R. Du, H. L. Stormer, D. C. Tsui, L. N. Pfeiffer, and K. W. West, Phys. Rev. Lett. 73, 3274 (1994).

[9] H. C. Manoharan, M. Shayegan, and S. J. Klepper, Phys. Rev. Lett. 73, 3270 (1994).

[10] V. J. Goldman, B. Su, and J. K. Jain, Phys. Rev. Lett. 72, 2065 (1994).

[11] X. Ying, V. Bayot, M. B. Santos, and M. Shayegan, Phys. Rev. B 50, 4969 (1994).

[12] I. V. Kukushkin, R. J. Haug, K. v. Klitzing, and K. Ploog, Phys. Rev. Lett. 72, 736 (1994).

[13] V. Bayot, E. Grivei, H. C. Manoharan, X. Ying, and M. Shayegan, Phys. Rev. B 52, R8621 (1995). 
[14] I. V. Kukushkin, R. J. Haug, K. v. Klitzing, and K. Ploog, Phys. Rev. B 51, 18045 (1995).

[15] S. S. Mandal and V. Ravishankar, Phys. Rev. B 54, 8688 (1996).

[16] S. S. Mandal and V. Ravishankar, Phys. Rev. B 54, 8699 (1996).

[17] D. Stein, K. v. Klitzing, and G. Weimann, Phys. Rev. Lett. 51, 130 (1983).

[18] C. Kallin and B. I. Halperin, Phys. Rev. B 30, 5655 (1984).

[19] J. P. Longo and C. Kallin, Phys. Rev. B 47, 4429 (1993).

[20] In Ref. [15], the Zeeman term includes CS gauge fields as well. Strictly speaking, the $g$ factor then should have been renormalized one. Here the $g$ factor is the bare value of the system. Thus only applied magnetic field is included in the Zeeman term.

[21] T. Nakajima and H. Aoki, Phys. Rev. Lett. 73, 3568 (1994).

[22] F. D. M. Haldane, in The Quantum Hall Effect, edited by R. E. Prange and S. M. Girvin, (Springer-Verlag, New York), 1987.

[23] S. L. Sondhi, A. Karlhede, S. A. Kivelson, and E. H. Rezayi, Phys. Rev. B 47, 16419 (1993).

[24] S. E. Barrett, G. Dabbagh, L. N. Pfeiffer, K. W. West, and R. Tyco, Phys. Rev. Lett. 74, 5112 (1995).

[25] A. Schmeller, J. P. Eisenstein, L. N. Pfeiffer, and K. W. West, Phys. Rev. Lett. 75, $4290(1995)$.

[26] E. H. Aifer, B. B. Goldberg, and D. A. Broido, Phys. Rev. Lett. 76, 680 (1996).

[27] X. G. Wu and S. L. Sondhi, Phys. Rev. B 51, 14725 (1995). 


\section{FIGURE CAPTIONS}

FIG. 1. $\Delta E_{1}(q)$ and $\Delta E_{2}(q)$ are plotted in the units of $e^{2} / \epsilon l_{0}$ against $q l_{0} \cdot q=\infty$ asymptotes are same for both the cases.

FIG. 2. (a) $\Delta E_{1}^{ \pm}(q)$ and (b) $\Delta E_{2}^{ \pm}(q)$ are plotted in the units of $e^{2} / \epsilon l_{0}$ against $q l_{0}$. The lines (c) and (d) are the respective $q=\infty$ asymptotes.

FIG. 3. (a) $\Delta E_{1}^{(1)-}(q)$, (b) $\Delta E_{2}^{(1)-}(q)$, and (c) $\Delta E_{1}^{(2)-}(q)$ are plotted in the units of $e^{2} / \epsilon l_{0}$ against $q l_{0}$. The lines (d), (e), and (f) are the respective $q=\infty$ asymptotes.

FIG. 4. The spin flip mode, corresponding to flipping the spin from down to up, $\Delta E_{1}^{+}(q)$ is shown in the unit of $e^{2} / \epsilon l_{0}$.

FIG. 5. The spin-wave mode is shown in the unit of $e^{2} / \epsilon l_{0}$ for $p_{\uparrow}=1, p_{\downarrow}=0$, with the omission of Zeeman energy.

FIG. 6. The spin-wave mode is shown in the unit of $e^{2} / \epsilon l_{0}$ for $p_{\uparrow}=2, p_{\downarrow}=1$, with the omission of Zeeman energy.

FIG. 7. Thick (thin) lines represent single particle Hartree-Fock (bare) Green's functions. The wiggly lines represent the Coulomb interaction. (a) The self energy $\Sigma_{n}^{r}$ for the particles of spin index $r$ in the $n$th Landau level. (b) The single particle Hartree-Fock Green's function $G_{n}^{r}$ for $n$th landau level and spin index $r$.

FIG. 8. $\alpha_{i}=\left(n_{i}, r_{i}\right)$ denotes collectively the Landau level index $n_{i}$ and the spin index $r_{i}$. Thick (thin) lines represent single particle Hartree-Fock (bare) Green's functions. The dashed lines represent the probes. (a) Response function $\chi^{a b}(\omega, \mathbf{q})$ is diagrammatically shown. The shaded portion represents the vertex correction due to the Coulomb interaction. (b) The vertex function $\Gamma_{n_{1} n_{2}}^{r_{1} r_{2}}(\omega, \mathbf{q})$ is diagrammatically shown. 


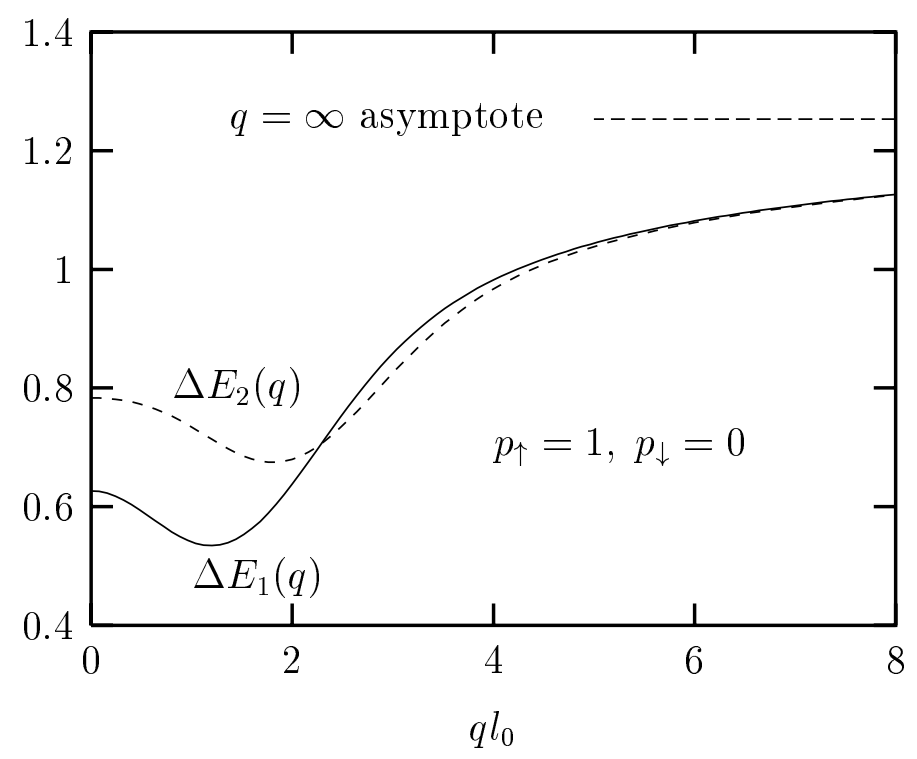

Fig.1 (Mandal) 


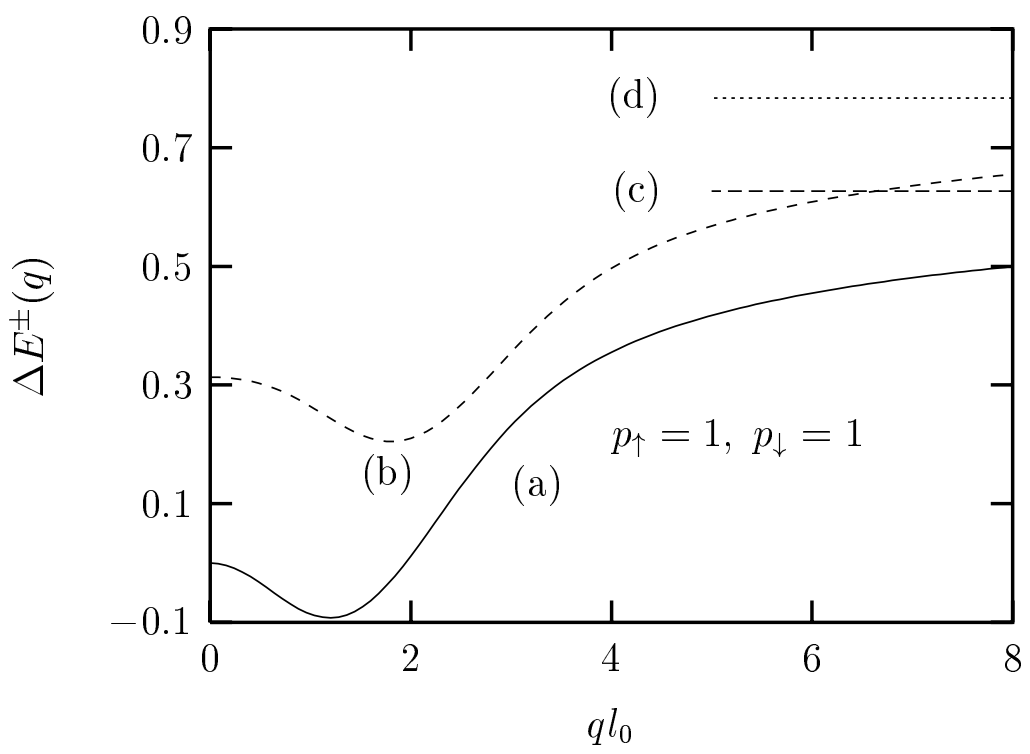

Fig.2 (Mandal) 


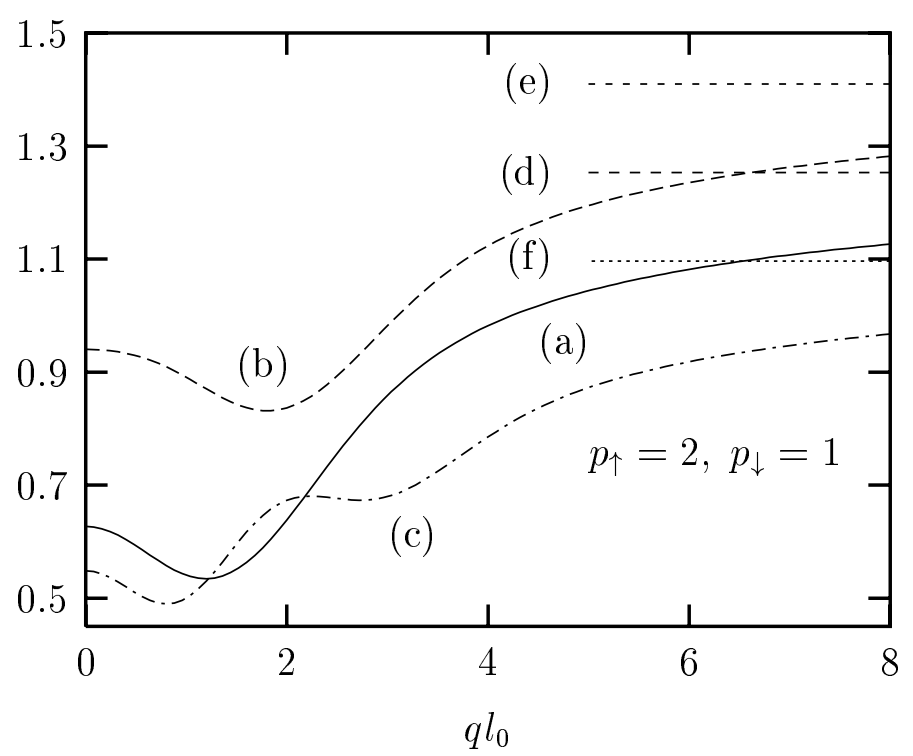

Fig.3 (Mandal) 


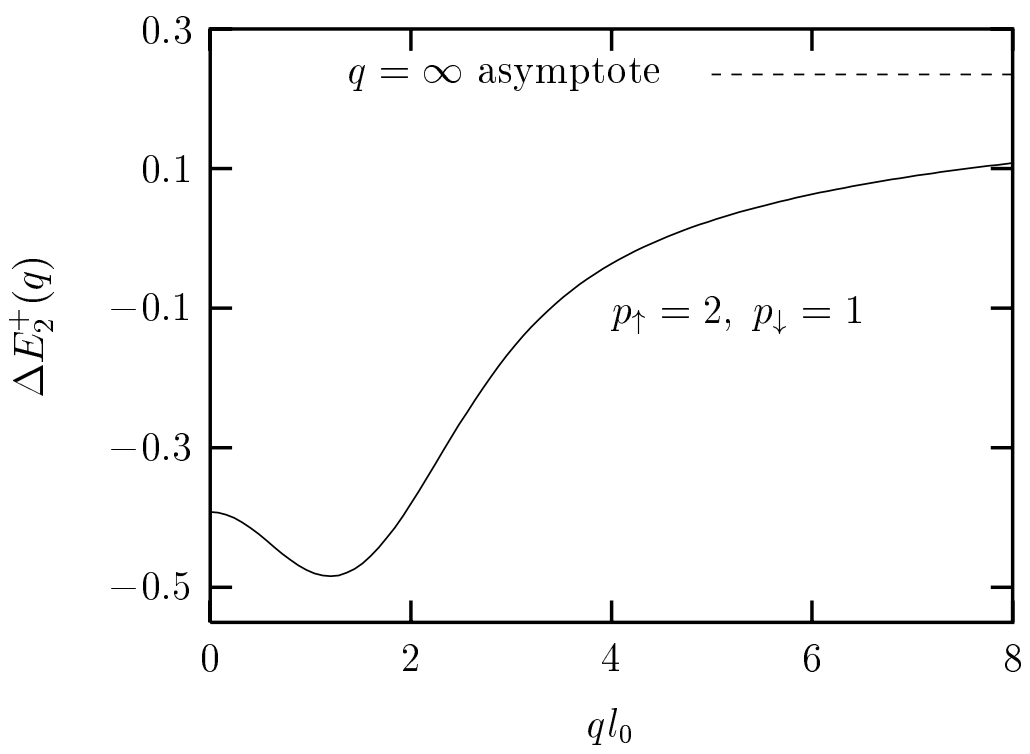

Fig.4 (Mandal) 


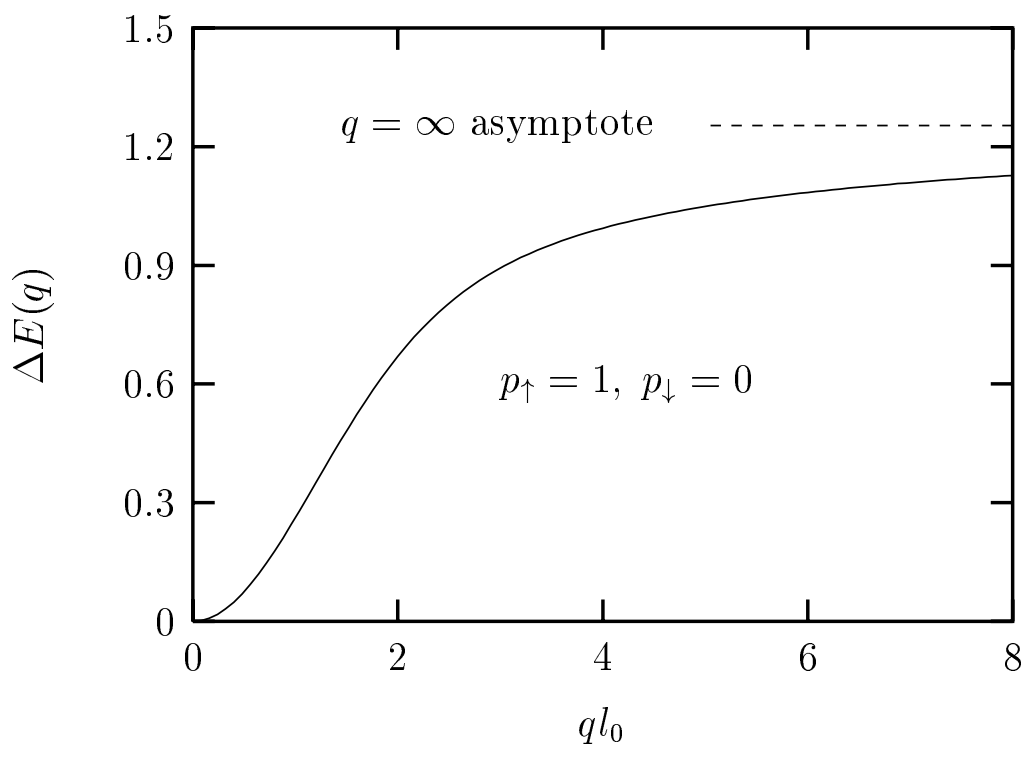

Fig.5 (Mandal) 


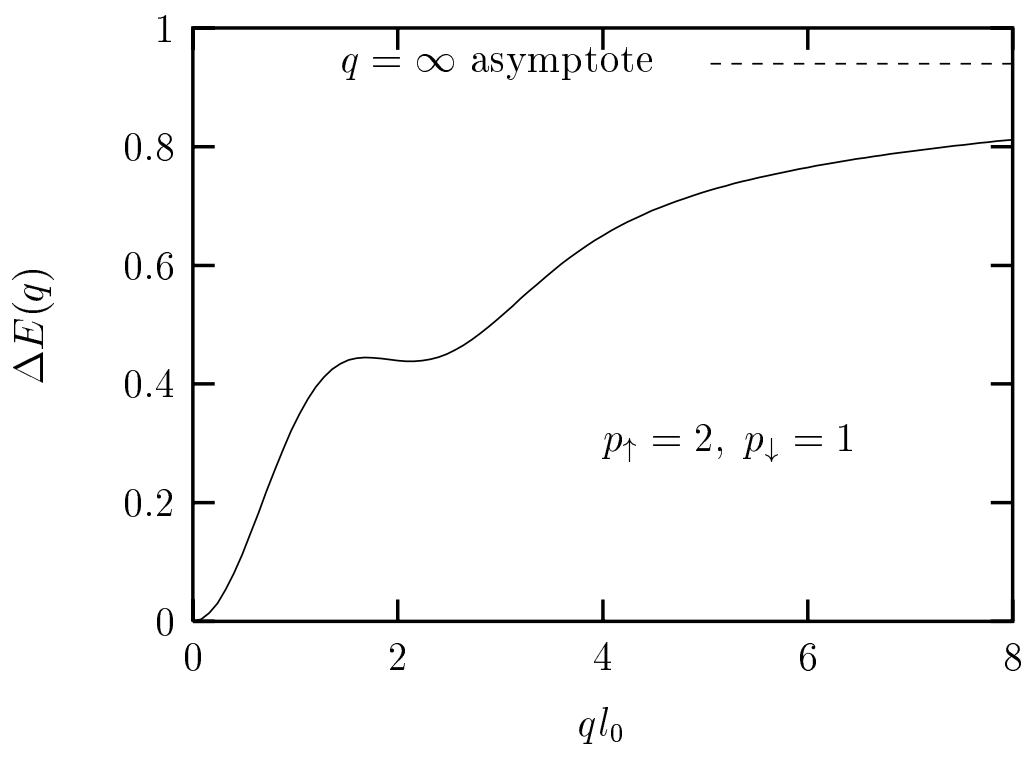

Fig.6 (Mandal) 


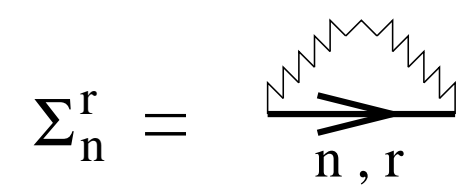

(a)

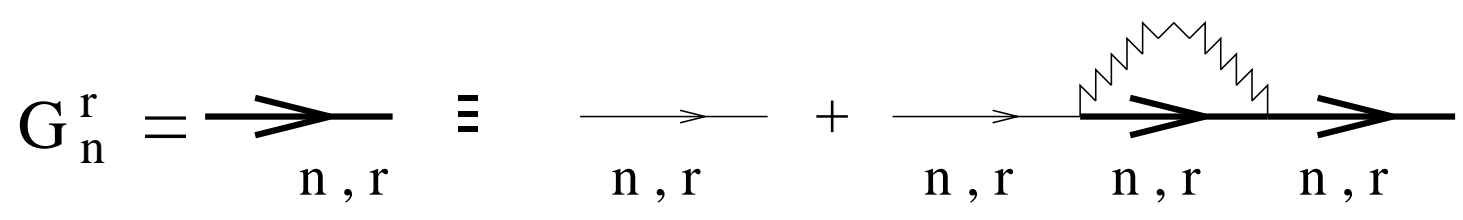

(b)

Fig.7 (Mandal) 


$$
\chi^{\mathrm{ab}}(\omega, \mathrm{q})=\underset{-\omega, \mathrm{q}}{\mathrm{a}}>_{\mathrm{r}_{2}}^{\mathrm{r}}
$$

(a)

$$
\Gamma_{\mathrm{n}_{1} \mathrm{n}_{2}}^{\mathrm{r}_{1} \mathrm{r}_{2}}(\omega, \mathrm{q})=\underbrace{\alpha_{\omega, \mathrm{q}}}_{\sum_{\alpha_{2}}^{\sum_{1}}} \equiv \sum_{\alpha_{\alpha_{2}}}^{\alpha_{1}}+\cdots, \mathrm{q}+
$$

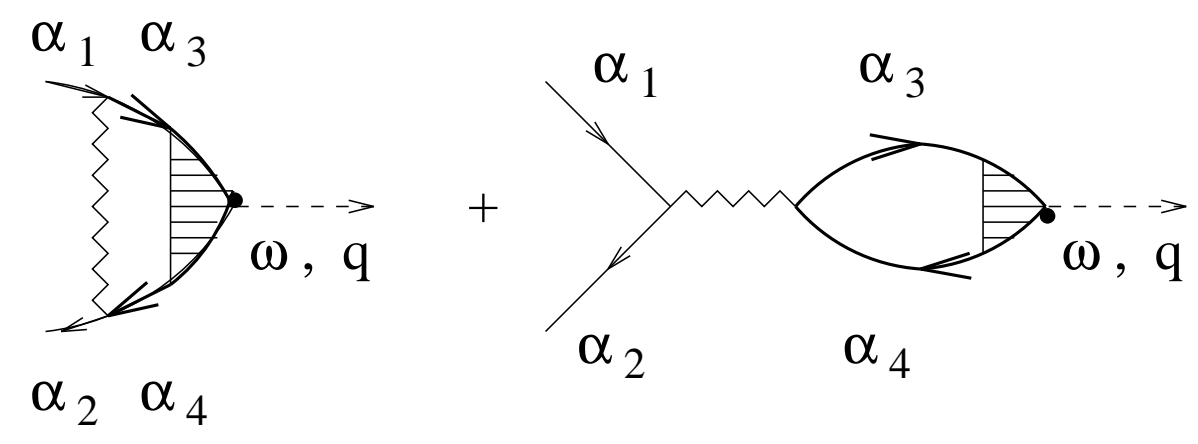

(b)

Fig.8 (Mandal) 